Gunnar Flötteröd, Kai Nagel

\title{
Some practical extensions to the cell transmission model
}

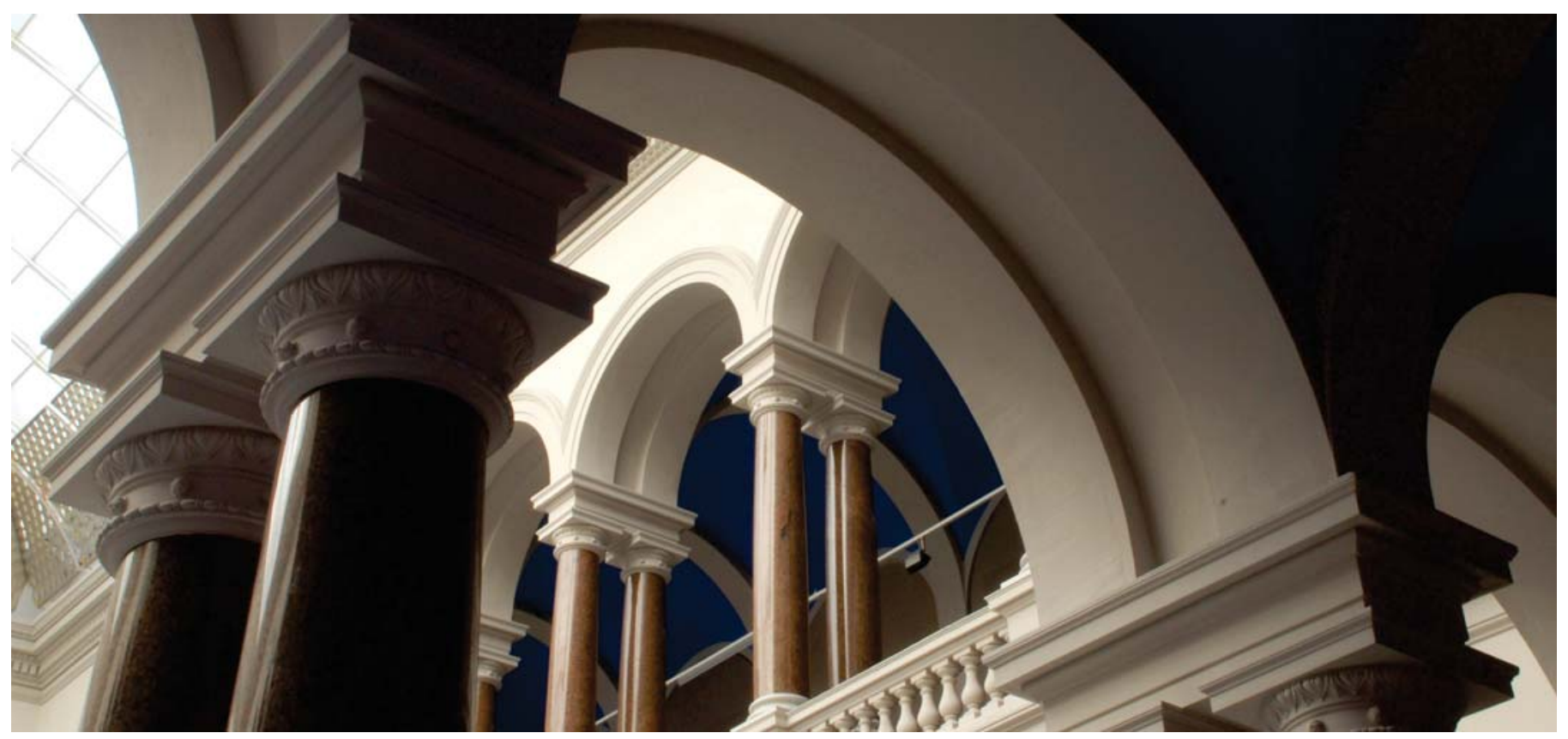

Flötteröd, G., Nagel, K.: Some practical extensions to the cell transmission model. In: 2005 IEEE Intelligent Transportation Systems Conference (ITSC), Vienna, Austria, 2005, p. 172-177. https://doi.org/10.1109/ITSC.2005.1520042 


\title{
Some practical extensions to the Cell Transmission Model
}

\author{
Gunnar Flötteröd* and Kai Nagel**
}

\begin{abstract}
This article describes some practical extensions to Daganzo's Cell Transmission Model. Flow calculations for straight, merge, and diverge cells are subsumed in a single computation scheme which allows for arbitrary cell connectivity. Since it is planned to apply the resulting model in a mathematical programming based traffic monitoring system, approximate sensitivities are also provided.
\end{abstract}

\section{INTRODUCTION}

Any intelligent transportation system's performance significantly depends on its capability to reproduce and predict the state of the traffic system under consideration. From a control engineering point of view, this can be achieved by representing the system in state space form and then applying an observation algorithm such as Kalman filtering [1], [10].

Pursuing this idea, we currently are in the process of setting up a traffic model with certain properties we found most appropriate in a state estimation context. Specifically, this article describes a network loading model, which is (like all other components of the overall system representation) a deterministic macroscopic discrete time model that can at least approximately be differentiated.

Although we sacrifice many benefits of microscopic approaches by choosing an aggregated model [2], [8], we still expect meaningful estimation results due to the straightforward applicability of a large number of mathematical programming algorithms to our problem formulation (as compared to the far more difficult handling of microscopic traffic simulator outputs) [3], [9]. Since we also work on agent based models, this work can be understood to complement such approaches.

To build on a strong foundation, we chose the Cell Transmission Model (CTM) as a starting point. It fulfills the aforementioned requirements and has proven to be a proper numerical approximation of the well-established LWR representation of traffic flow [4], [7]. Although it was originally proposed for highway traffic, we intend to apply it also to urban systems, where traffic intrinsic dynamics become secondary compared to the effect of light signals. In this case, even simpler models appear to be sufficient to capture relevant effects [6]. Still, the possibility for seamless transition from freeway to urban traffic using a single model makes the CTM even more attractive. In addition, it is a first order model with an accordingly low number of states and parameters to be estimated.

\footnotetext{
*Gunnar Flötteröd is with the Group for Transport Systems Planning and Transport Telematics, Technische Universität Berlin, D-10587 Berlin, Germany. Tel: +49-30-314-29520, fax: +49-30-314-26269, mail: floetteroed@vsp.tu-berlin.de.

**Kai Nagel is head of the Group for Transport Systems Planning and Transport Telematics, mail: nagel@vsp.tu-berlin.de.
}

We found that some extensions to the CTM would be of use for practical application: While the original CTM poses several restrictions on cell linkage [5], this paper discusses a generalized connection structure which includes the original straight, merge and diverge cells as special cases. ${ }^{1}$ This allows to directly map road networks of arbitrary topology onto the Extended Cell Transmission Model (ECTM).

In order to unify these extensions and to improve our implementation's robustness, we cast them into a formal framework representing all possible flow calculations in terms of a general dynamic process. Since sensitivity analysis for this process is provided, the entire ECTM also is (approximately) differentiable.

The remainder of this article is organized as follows: Section II describes a general process of resource consumption, from which all flow calculation equations of the ECTM can be derived. Section III shows how the original CTM is represented by this process and describes extended modeling capabilities. Section IV provides approximate sensitivity analysis and section $\mathrm{V}$ gives an outlook on further works.

\section{A General Process of Resource Consumption}

This section is not necessarily traffic-specific. It discusses a general process of resource consumption. Considering traffic flow modeling, these resources correspond to available vehicles on an upstream road segment and the available space on an according downstream segment. For readability, approximate sensitivity analysis is postponed until section IV.

We consider an $n$-dimensional process with time index $k=0,1, \ldots, K$. Every state $x_{i}^{(k)}$ (element of state vector $\left.\mathbf{x}^{(k)} \in \mathbb{R}^{n}, i=1 \ldots n\right)$ can be considered as some resource which is used up during the process. Its rate of consumption equals a nonnegative and finite flow rate $f_{i}^{(k)} \geq 0$, which remains constant within every time step. Denote a time step $k$ 's duration by $t^{(k)}$. The process' evolvement is then given by $x_{i}^{(k+1)}=x_{i}^{(k)}-f_{i}^{(k)} t^{(k)}$.

Any state that has run dry (i.e. reached zero) is excluded from further calculations. The set $\mathcal{D}^{(k)}=\left\{i ; x_{i}^{(k)}>0\right\}$ contains all states still relevant in time step $k$. Because of the states' non-negativity constraints, $t^{(k)} \leq x_{i}^{(k)} / f_{i}^{(k)}$ must hold for all $i \in \mathcal{D}^{(k)}$. The maximum duration of time step $k$ constrained exclusively by state $i \in \mathcal{D}^{(k)}$ (also called the availability of resource $i$ within time step $k$ ) is given by

\footnotetext{
${ }^{1}$ It may be argued that arbitrary connectivity can also be achieved by proper combination of the three basic cell types described in [5]. Still, this inevitably imposes possibly unwanted dependencies between flows through such settings, and requires somewhat involved construction of more complicated intersections.
} 


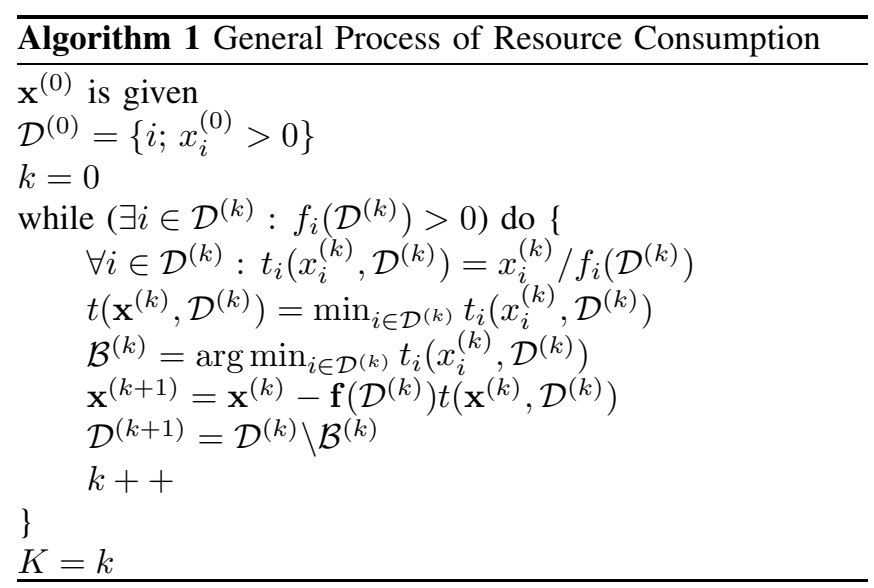

$t_{i}^{(k)}=x_{i}^{(k)} / f_{i}^{(k)} \in(0, \infty)$. It is assumed that every time step $k$ lasts until at least one state in $\mathcal{D}^{(k)}$ has reached its minimal (zero) value. (Zero flow rates may imply infinite availabilities for certain states, which is feasible.) Time step $k$ 's duration can now be given by $t^{(k)}=\min _{i \in \mathcal{D}^{(k)}} t_{i}^{(k)}$. Because $t_{i}^{(k)}$ is well defined and strictly positive for all $i \in \mathcal{D}^{(k)}$, this implies a likewise strictly positive duration $t^{(k)}$ of all time steps.

For convenience, a set $\mathcal{B}^{(k)}=\arg \min _{i \in \mathcal{D}^{(k)}} t_{i}^{(k)}$ is defined. ${ }^{2}$ It contains those state indices having run dry at the end of time step $k$. Therefore, we can give $\mathcal{D}^{(k+1)}=$ $\mathcal{D}^{(k)} \backslash \mathcal{B}^{(k)}$ as an update equation. Flow rates $f_{i}^{(k)}$ depend only on the set $\mathcal{D}^{(k)}$ of currently available states and do not vary with respect to the time index otherwise: $f_{i}^{(k)}=$ $f_{i}\left(\mathcal{D}^{(k)}\right)$. From this it also results that $t_{i}^{(k)}=t_{i}\left(x_{i}^{(k)}, \mathcal{D}^{(k)}\right)$. Since all consumption rates of a given time step $k$ are defined by $\mathcal{D}^{(k)}$, we phrase that "time step $k$ is under regime $\mathcal{D}^{(k)}$ ". The process configuration given so far implies that $f_{i}\left(\mathcal{D}^{(k)}\right)=0$ for all states $i \notin \mathcal{D}^{(k)}$. Availabilities of these states are neither defined nor needed. The process terminates when all flows have ceased, i.e. $f_{i}\left(\mathcal{D}^{(K)}\right)=0 \forall i \in \mathcal{D}^{(K)}$. Algorithm 1 gives an overview.

It has to be pointed out that the temporal aspect of this process is not to be interpreted physically: One entire process run corresponds to a single vehicular flow rate calculation for a set of adjacent cells within one simulation time step. Therefore, only the final state reached by the process is of relevance to the physical simulation. ${ }^{3}$

\section{An Extended Cell Transmission Model}

In this section, the flow calculation equations of the original CTM cell types are expressed in terms of the general process given in Section II. Several extensions to the model are discussed.

\section{A. Preliminaries}

The ECTM is to be applied in a real time state estimation context. Therefore, simplicity for fast numerical

\footnotetext{
${ }^{2}$ The argmin function returns the set of all minimizing indices.

${ }^{3}$ This terminology will be maintained throughout the article: One simulation time step for calculation of vehicular flow rates consists of a complete run of the dynamic (sub-)process, which itself contains of a number of further sub-time steps in which (resource) consumption rates are evaluated.
}

treatment and sufficiently detailed modeling capabilities have to be balanced: Although the ECTM operates in destinationoriented mode, all flow calculations on cell level are based only on local information such as turning proportions. This is achieved by appropriate aggregation and disaggregation procedures at the beginning and end of every simulation time step. These simplifications have some influence on the ECTM's precision: All destination oriented properties are equally distributed within a cell. Therefore, the precision of FIFO compliance is not in the order of a simulation time step (as in the original model), but rather in the order of a congested cell's traversal time.

In the original model, the road network is represented by a graph comprised of vertices corresponding to cells and edges describing the linkage between them. Different cell types for different connection types exist. In our implementation of the ECTM, the vertex set is augmented by so-called connectors, being responsible for flow calculations. The cells themselves are reduced to occupancy containers with little internal functionality. The only connectivity constraint is given by the need for alternating sequences of cells and connectors.

\section{B. Representation of physical traffic states in the general process}

Before different connector types can be discussed, the relationships between state variables of a real traffic network and those of the general process described above are given.

The state of a cell $i$ is (as in the original CTM) given by its occupancy $\xi_{i} \cdot{ }^{4}$ We denote its maximal occupancy by $\hat{\xi}_{i}$ and its maximal vehicular flow rate by $\hat{\varphi}_{i}$. Cell geometry constraints are the same as in the original CTM.

The duration of a simulation time step is $\theta$. It starts with a sweep over all connectors for flow calculations. Since this implies a run of one sub-process for every connector $C$, an initial state $\mathbf{x}^{(0)}$ for such a process has to be provided. This is achieved by letting $x_{P_{i}}^{(0)}=\min \left(\xi_{P_{i}}, \hat{\varphi}_{P_{i}} \theta\right) ; P_{i} \in P(C)$ and $x_{S_{j}}^{(0)}=\min \left(\hat{\xi}_{S_{j}}-\xi_{S_{j}}, \hat{\varphi}_{S_{j}} \theta\right) ; S_{j} \in S(C)$ with $P(C)$ $(S(C)$ ) being the set of cells preceeding (succeeding) $C$ (see figs. 1 and 2). This initial reduction of the maximal available resources incorporates (again, as in the original CTM) flow rate constraints into the sub-process.

\section{Straight Flows}

The CTM's basic flow calculation rule states that the number of transmitted vehicles between two succeeding cells equals the minimum of available vehicles upstream, available space downstream, and an upper flow constraint. It holds in general that the CTM always maximizes flows with respect to given upstream (vehicle availability, turning proportions), downstream (space availability, inflow priorities) and flow rate constraints.

Straight connectors of the ECTM have exactly one predecessor and one successor cell. Speaking in terms of the

\footnotetext{
${ }^{4}$ As pointed out, destination oriented properties are not relevant at cell level. Sources and sinks are not modeled. In this article, we only consider traffi c being already on the network.
} 
general process, the state vector $\mathbf{x}=\left(\begin{array}{ll}x_{P} & x_{S}\end{array}\right)^{T}$ is twodimensional: $x_{P}$ represents the number of available upstream vehicles on predecessor cell $P$, whereas $x_{S}$ equals the available space on successor cell $S$ as defined in section III-B.

Since flow conservation holds, vehicles leaving the predecessor equally reduce the space left on the successor cell. This is expressed in the resource consumption vector $\mathbf{f}(\{P, S\})=\left(\begin{array}{ll}1 & 1\end{array}\right)^{T}$, which corresponds to the only regime $\{P, S\}$ with a nonzero consumption rate. This regime's duration is $\min \left(x_{P}, x_{S}\right)$. Multiplying this duration with the consumption rate of 1 for either involved cell yields the same flow calculation rule as stated above for the original CTM.

\section{Merge Flows}

The original CTM allows for merge connections between exactly two predecessor cells and one successor cell. The flow calculation rules state that both predecessors are allowed to send all their available vehicles as long as these can be accepted by the successor cell. If this is not the case, the successor's available space is shared between the predecessors in a ratio according to their priorities $\alpha_{1} \in[0,1]$ and $\alpha_{2}=1-\alpha_{1}$. If this causes all available vehicles of one predecessor to be removed but still leaves available capacity on the successor, this space is filled up as far as possible with vehicles from the complementary predecessor.

In terms of the general process, the initial state vector is given by $\mathbf{x}=\left(\begin{array}{lll}x_{P_{1}} & x_{P_{2}} & x_{S}\end{array}\right)^{T}$, where $x_{P_{1}}$ and $x_{P_{2}}$ denote the available vehicles on the predecessors and $x_{S}$ equals the available space on the successor cell. The process' evolution then is fully defined by the following non-zero regime dependent consumption rate vectors: $\mathbf{f}\left(\left\{P_{1}, P_{2}, S\right\}\right)=\left(\begin{array}{lll}\alpha_{1} & \alpha_{2} & 1\end{array}\right)^{T}, \mathbf{f}\left(\left\{P_{1}, S\right\}\right)=\left(\begin{array}{lll}1 & 0 & 1\end{array}\right)^{T}$ and $\mathbf{f}\left(\left\{P_{2}, S\right\}\right)=\left(\begin{array}{lll}0 & 1 & 1\end{array}\right)^{T}$. Inspection of the only possible regime sequences $\left\{P_{1}, P_{2}, S\right\} \rightarrow\left\{P_{1}, S\right\}$ and $\left\{P_{1}, P_{2}, S\right\} \rightarrow\left\{P_{2}, S\right\}$ shows that this setup yields identical behavior as the original CTM.

General merge connectors as shown in the upper part of fig. 1 have an arbitrary number of $I \geq 2$ predecessor cells and exactly one successor cell. The first $I$ resources comprising the resulting state vector are the available vehicles $x_{P_{i}}$ on predecessor cells $i=1, \ldots, I$. The available space $x_{S}$ on the successor cell makes up one additional resource: $\mathbf{x}=\left(\begin{array}{llll}x_{P_{1}} \ldots & x_{P_{I}} x_{S}\end{array}\right)^{T}$. A natural extension of the aforementioned priority dependent flow calculation scheme can now be defined by: ${ }^{5}$

$$
\begin{aligned}
\mathbf{f}(\mathcal{D}) & =\left(f_{P_{1}}(\mathcal{D}) \ldots f_{P_{I}}(\mathcal{D}) \sum_{i=1}^{I} f_{P_{i}}(\mathcal{D})\right)^{T} \\
f_{P_{i}}(\mathcal{D}) & =\left\{\begin{array}{cl}
\alpha_{i} & P_{i} \in \mathcal{D} \wedge S \in \mathcal{D} \\
0 & \text { otherwise. }
\end{array}\right.
\end{aligned}
$$

\footnotetext{
${ }^{5}$ Obviously, priorities do not have to sum up to 1 . Still, some care has to be taken, if only zero priority predecessors remain in $\mathcal{D}$. In this case, any strictly positive and among elements of $\mathcal{D}$ equally distributed priorities solve the problem.
}

Fig. 1. Merge and Diverge Connectors

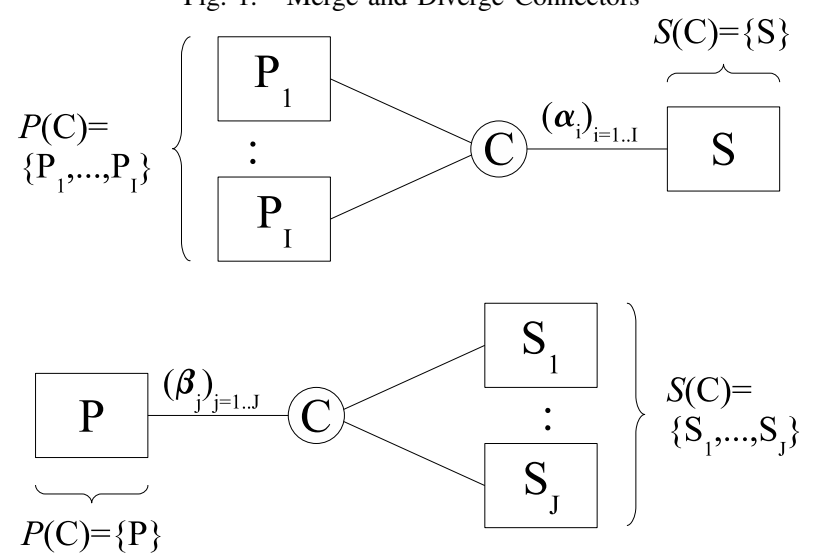

Fig. 2. General Connector

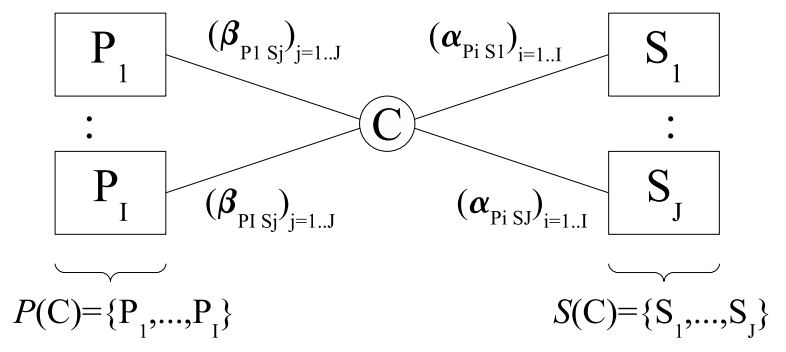

For $I=2$ this equals the original CTM's merge transmission rules.

\section{E. Diverge Flows}

Diverges of the original CTM split one predecessor cell into exactly two successor cells. In non-destination oriented mode, splitting proportions are given by coefficients $\beta_{1} \in$ $[0,1]$ and $\beta_{2}=1-\beta_{1}$. In this case, state vector $\mathbf{x}=$ $\left(\begin{array}{lll}x_{P} & x_{S_{1}} & x_{S_{2}}\end{array}\right)^{T}$ is comprised of predecessor $P$ 's available vehicles and the available space on successors $S_{1}$ and $S_{2}$. Allowing for only one non-zero consumption rate vector $\mathbf{f}\left(\left\{P, S_{1}, S_{2}\right\}\right)=\left(\begin{array}{lll}1 & \beta_{1} & \beta_{2}\end{array}\right)^{T}$ implies the assumption of exactly one lane on the predecessor cell: If a vehicle at the head of the queue on this lane is unable to enter its successor cell, it completely blocks the diverge. The total outflow from $P$ is then given by $\min \left(x_{P}, x_{S_{1}} / \beta_{1}, x_{S_{2}} / \beta_{2}\right)$, just as for the original CTM.

A generalization of this to more than two successors (see lower part of fig. 1) can be easily achieved by introduction of an extended state vector $\mathbf{x}=\left(x_{P} x_{S_{1}} \ldots x_{S_{J}}\right)^{T}$ and an according flow vector

$$
\mathbf{f}\left(\left\{P, S_{1}, \ldots, S_{J}\right\}\right)=\left(1 \beta_{1} \ldots \beta_{J}\right)^{T}
$$

for the only non-zero consumption regime.

\section{F. General Intersection Flows}

Merges and diverges are now to be combined in a general intersection connector (see fig. 2). Although an appealing solution would be to directly combine both calculation 
schemes, the number of flow constraints ( $I J$ turning proportions and $I J$ priorities) is larger than the number of available degrees of freedom ( $I J$ predecessor-successor consumption rates). Therefore, some simplifications are necessary.

As it has been pointed out before, turning proportions fully subsume destination oriented flow behavior. Therefore, they should be implemented without error: For every predecessor $P_{i} \in \mathcal{D}$ a consumption rate $f_{P_{i}}(\mathcal{D})$ is defined, from which successor oriented consumption rates $f_{P_{i} S_{j}}(\mathcal{D})=\beta_{P_{i} S_{j}} f_{P_{i}}(\mathcal{D})$ are obtained for all $P_{i}, S_{j} \in \mathcal{D}$. This ensures $f_{P_{i} S_{j}}(\mathcal{D}) / f_{P_{i} S_{l}}(\mathcal{D})=\beta_{P_{i} S_{j}} / \beta_{P_{i} S_{l}}$ for all $P_{i}, S_{j}, S_{l} \in \mathcal{D}$. A weighted-average priority $\alpha_{P_{i}}(\mathcal{D})=$ $\sum_{S_{j} \in \mathcal{D}} \beta_{P_{i} S_{j}} \alpha_{P_{i} S_{j}}$ is now employed to state a simplified priority rule $f_{P_{i}}(\mathcal{D}) / f_{P_{l}}(\mathcal{D})=\alpha_{P_{i}}(\mathcal{D}) / \alpha_{P_{l}}(\mathcal{D})$ for all nonblocked $P_{i}, P_{l} \in \mathcal{D}$. This is ensured by letting $f_{P_{i}}(\mathcal{D})=$ $\alpha_{P_{i}}(\mathcal{D})$ for all $P_{i} \in \mathcal{D}$ that are not blocked by an unavailable successor $S_{j} \notin \mathcal{D}$ towards which a positive turning fraction $\beta_{P_{i} S_{j}}$ exists. The full resource state vector $\mathbf{x}=$ $\left(x_{P_{1}} \ldots x_{P_{I}} x_{S_{1}} \ldots x_{S_{J}}\right)^{T}$ is then consumed by

$$
\begin{aligned}
\mathbf{f}(\mathcal{D}) & =\left(f_{P_{1}}(\mathcal{D}) \ldots f_{P_{I}}(\mathcal{D}) f_{S_{1}}(\mathcal{D}) \ldots f_{S_{J}}(\mathcal{D})\right)^{T} \\
f_{P_{i}}(\mathcal{D}) & =\left\{\begin{array}{cl}
\sum_{S_{j} \in \mathcal{D}} \beta_{P_{i} S_{j}} \alpha_{P_{i} S_{j}} & P_{i} \in \mathcal{D} \wedge \mathcal{D} \supset \\
0 & \begin{array}{l}
\left\{S_{j} ; \beta_{P_{i} S_{j}}>0\right\} \\
\text { otherwise }
\end{array} \\
f_{S_{j}}(\mathcal{D}) & =\sum_{P_{i} \in \mathcal{D}} \beta_{P_{i} S_{j}} f_{P_{i}}(\mathcal{D}) .
\end{array}\right.
\end{aligned}
$$

The possibility to easily model quite general intersections by this scheme is illustrated in fig. 3 .

The general connector comprises all previously defined connector types, as can be seen from choosing $I=1$ and/or $J=1$. Therefore, all properties of this general connector also hold for more specific connector types. In addition, this connector's behavior is identical to the original CTM whenever the set of available resources (even in a general $I$ on $J$ setting) reduces to constellations already defined in the CTM.

\section{Approximate Sensitivity Analysis}

In this section, an approximate Jacobian $\partial \mathbf{x}^{(K)} / \partial \mathbf{x}^{(0)}$ for the initial state $\mathbf{x}^{(0)}$ 's mapping onto the final state $\mathbf{x}^{(K)}$ resulting from the process described in Algorithm 1 is determined. Because of the general process' temporal multistage property, the problem can be solved by a recursive update scheme which mainly requires the calculation of $\partial \mathbf{x}^{(k+1)} / \partial \mathbf{x}^{(k)}$ in every sub-time step $k^{6}$

The results given in this section are not necessary to conduct simulations with the ECTM. They allow for a linearization of the model, as it is useful e.g. for state estimation purposes.

\section{A. Preliminaries}

The approximate Jacobian is comprised of the directional derivatives along all coordinate axes. When evaluated at

\footnotetext{
${ }^{6}$ Operators will be used as follows: $\frac{\partial}{\partial}$ denotes partial derivatives as well as components of the approximate Jacobian. $\delta$ denotes a small variation.
}

Fig. 3. Exemplary intersection modeling

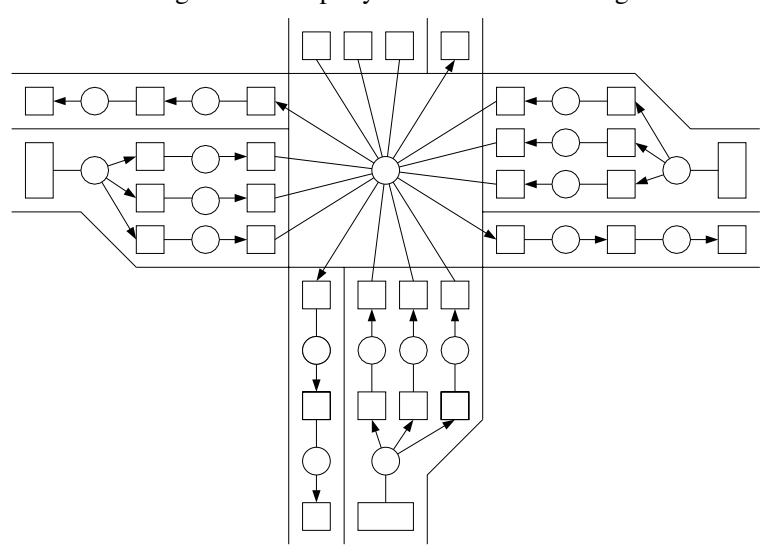

A general intersection consists of several legs with every leg being comprised of a certain number of entering and exiting lanes. Within longer road segments, lanes are not explicitely represented. When approaching the intersection, lane choice can be modeled by a single diverge connector. The intersection itself is represented by a single general connector, which distributes traffic flows according to turning proportions and priorities. (Circles denote connectors and boxes denote cells. Where no flow direction is indicated by an arrow, it goes from cell to connector.)

points of non-smoothness, an average of left and right sided sensitivities is used. ${ }^{7}$ As the following analysis will show, small state variations before a certain sub-time step may cause intermediate regimes around this sub-time step to occur. Since we are interested in averaged sensitivities around every sub-time step, we replace the original process by one shifted half a sub-time step before calculating sensitivities. The shifted process starts at $\mathbf{x}^{(-1 / 2)}=\mathbf{x}^{(0)}$ and runs until sub-time step $K+1 / 2$ :

$$
\begin{aligned}
\mathbf{x}^{(1 / 2)}= & \mathbf{x}^{(-1 / 2)}+\mathbf{f}\left(\mathcal{D}^{(0)}\right) t\left(\mathbf{x}^{(0)}, \mathcal{D}^{(0)}\right) / 2 \\
\mathbf{x}^{(k+1 / 2)}= & \mathbf{x}^{(k-1 / 2)} \\
& +\mathbf{f}\left(\mathcal{D}^{(k-1)}\right) t\left(\mathbf{x}^{(k-1)}, \mathcal{D}^{(k-1)}\right) / 2 \\
& +\mathbf{f}\left(\mathcal{D}^{(k)}\right) t\left(\mathbf{x}^{(k)}, \mathcal{D}^{(k)}\right) / 2 \\
\mathbf{x}^{(K+1 / 2)}= & \mathbf{x}^{(K-1 / 2)} \\
& +\mathbf{f}\left(\mathcal{D}^{(K-1)}\right) t\left(\mathbf{x}^{(K-1)}, \mathcal{D}^{(K-1)}\right) / 2
\end{aligned}
$$

Apart from this formal shift in time this process is identical to the original one. Its result $\mathbf{x}^{(K+1 / 2)}$ is generated just as $\mathbf{x}^{(K)}$, therefore the shifted Jacobian $\partial \mathbf{x}^{(K+1 / 2)} / \partial \mathbf{x}^{(-1 / 2)}$ equals the original $\partial \mathbf{x}^{(K)} / \partial \mathbf{x}^{(0)}$.

Some additional remarks are necessary for the following discussion:

1) The relationship between any state and its availability

\footnotetext{
${ }^{7}$ Of course, this simplifi cation has to be kept in mind when applying a certain algorithm to the complete state estimation problem.
} 
is strictly monotonous for all $i \in \mathcal{D}^{(k)}$ :

$$
\frac{\partial t_{i}\left(x_{i}^{(k)}, \mathcal{D}^{(k)}\right)}{\partial x_{i}^{(k)}}=\frac{1}{f_{i}\left(\mathcal{D}^{(k)}\right)}>0 .
$$

2) Every state running dry at the end of sub-time step $k$ had a positive flow rate during this sub-time step:

$$
j \in \mathcal{B}^{(k)} \Rightarrow f_{j}\left(\mathcal{D}^{(k)}\right)>0 .
$$

(Otherwise, the state could not have run dry.)

3) A small increase of any resource $j \in \mathcal{B}^{(k)}$ having just run dry may increase this resource's availability by more than an equally small value because of a zero flow rate $f_{j}\left(\mathcal{D}^{(k+1)} \cup\{j\}\right)=0$. In this case, the remaining process must not be influenced by this variation in terms of zero sensitivities with respect to the varied resource. This requirement is fulfilled for the general connector defined in III-F: If $j$ corresponds to a cell preceeding the connector, $j \in \mathcal{B}^{(k)}$ implies that the according cell is empty after sub-time step $k$. If now an additional car is put in this cell (increase of resource $j$ ), and this vehicle is unable to enter any succeeding cell, it still does not influence any other vehicles, since it is the only one on its cell. If $j$ corresponds to a cell succeeding the connector, $j \in \mathcal{B}^{(k)}$ implies that the according cell is totally blocked after sub-time step $k$. If now the last car in this cell is removed (increase of resource $j$ ), the new space on $j$ remains unused only if the remaining available upstream resources have a zero turning proportion towards this successor. Still, this also implies that no predecessor outflow rates are influenced by this change on $j$. From this informal argumentation $^{8}$ it becomes clear, that variations of such resources can be treated independently of the remaining process variables. Therefore, they will not be explicitely discussed in the succeeding sensitivity analysis and only the more difficult case

$$
j \in \mathcal{B}^{(k)} \Rightarrow f_{j}\left(\mathcal{D}^{(k+1)} \cup\{j\}\right)>0
$$

will be considered. This requirement is always fulfilled if $j$ is the only element in $\mathcal{B}^{(k)}$, since this implies $\mathcal{D}^{(k+1)} \cup\{j\}=\mathcal{D}^{(k)}$ and therefore (2) holds.

4) If state $j$ is the only element in $\mathcal{B}^{(k)}$, regime $\mathcal{D}^{(k)}$ 's duration is limited only by $j$ 's availability, i.e. $t^{(k)}=$ $t_{j}^{(k)}$. Using (1) and (2), a small variation $\delta x_{j}^{(k)}$ of state $j$ implies the following small variation $\delta t^{(k)}$ of regime $\mathcal{D}^{(k)}$ 's duration:

$$
\mathcal{B}^{(k)}=\{j\} \Rightarrow \delta t^{(k)}=\frac{\delta x_{j}^{(k)}}{f_{j}\left(\mathcal{D}^{(k)}\right)} .
$$

\section{B. Initial Variation}

In the first sub-time step of the shifted process a transition from $k=-1 / 2$ to $1 / 2$ is examined. $\mathcal{B}^{(-1)}=$ $\{1, \ldots, n\} \backslash \mathcal{D}^{(0)}$ consolidates those resources that are already exhausted when the process starts. $\mathcal{D}^{(-1)}=\{1, \ldots, n\}$ equals the set of all possible state indices.

\footnotetext{
${ }^{8}$ The equations in III-F can be employed to support this more formally.
}

Fig. 4. Initial variation.

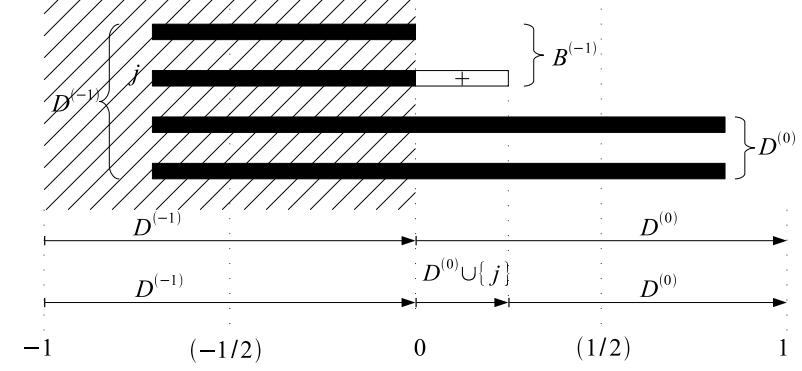

Schematic representation of resource variation at the process beginning. Bold lines represent states' availabilities. Below, the assignment of these resources to time steps and their according regimes is shown. The shaded area covers the hypothetical time span before the process actually starts.

From Fig. 4 the nonzero dependencies for an initial variation can be identified: All states $i \notin \mathcal{D}^{(0)}$ stay zero for the entire time span of regime $\mathcal{D}^{(0)}$ and remain uninfluenced by all other states. If they are perturbed themselves by an infinitely small value $\delta x_{i}^{(-1 / 2)}>0$, there always remains enough time for them to run dry again (with a positive consumption rate ensured by (3)) before sub-time step $1 / 2$ is reached.

States $i \in \mathcal{D}^{(0)}$ run dry not earlier than in sub-time step 1 and have a constant consumption rate. Therefore, they have a strictly positive value at sub-time step $1 / 2$. Considering the general state equation, to affect $x_{i}^{(1 / 2)}$, either the preceeding value $x_{i}^{(-1 / 2)}$ or the consumption rate $f_{i}\left(\mathcal{D}^{(0)}\right)$ have to be perturbed. Since the consumption rate is fully defined by regime $\mathcal{D}^{(0)}$ 's composition, only variations of states $j \in$ $\mathcal{B}^{(-1)}$ (hypothetically ending directly before regime $\mathcal{D}^{(0)}$ starts) can influence it.

1) $\boldsymbol{i}=\boldsymbol{j} \in \mathcal{D}^{(0)}$. Any variation $\delta x_{i}^{(-1 / 2)}$ of $x_{i}^{(-1 / 2)}$ directly punches through to the next sub-time step without influencing any other states:

$$
\delta x_{i}^{(1 / 2)}=\delta x_{i}^{(-1 / 2)} .
$$

2) $i \in \mathcal{D}^{(0)}, j \in \mathcal{B}^{(-1)}$. Since $x_{j}^{(-1 / 2)}=0$, only a positive variation $\delta x_{j}^{(-1 / 2)}>0$ is feasible. Such a variation implies by (1) an increase in state $j$ 's availability and causes a new regime $\mathcal{D}^{\prime}=\mathcal{D}^{(0)} \cup\{j\}$ to occur at the very beginning of $\mathcal{D}^{(0)}$ (see Fig. 4). $\mathcal{D}^{\prime}$ is limited by $\mathcal{B}^{\prime}=\{j\}$, therefore (4) can be used to obtain its duration $\delta t^{\prime}=\delta x_{j}^{(-1 / 2)} / f_{j}\left(\mathcal{D}^{\prime}\right)$. During $\delta t^{\prime}$, all states $i \in \mathcal{D}^{(0)}$ are reduced by flow rates $f_{i}\left(\mathcal{D}^{\prime}\right)$ instead of $f_{i}\left(\mathcal{D}^{(0)}\right)$. This varies the subsequent $x_{i}^{(1 / 2)}$ by $\delta x_{i}^{(1 / 2)}=\left(f_{i}\left(\mathcal{D}^{(0)}\right)-f_{i}\left(\mathcal{D}^{\prime}\right)\right) \delta t^{\prime}$. Therefore, we obtain

$$
\frac{\delta x_{i}^{(1 / 2)}}{\delta x_{j}^{(-1 / 2)}}=\frac{f_{i}\left(\mathcal{D}^{(0)}\right)-f_{i}\left(\mathcal{D}^{(0)} \cup\{j\}\right)}{f_{j}\left(\mathcal{D}^{(0)} \cup\{j\}\right)}
$$

with a nonzero denominator ensured by (3). 
Fig. 5. Intermediate variation.

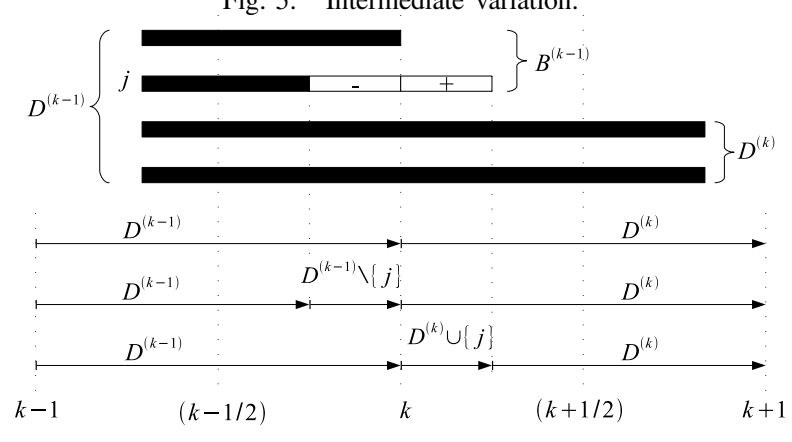

Schematic representation of intermediate resource variation. For explanations, see Fig. 4.

\section{Intermediate and Final Variation}

Following the outline given in the previous section, two cases can be identified in Fig. 5, for which non-zero sensitivities occur.

1) $i=j \in \mathcal{D}^{(k)}$. This is the punch-through-case as explained before:

$$
\delta x_{i}^{(k+1 / 2)}=\delta x_{i}^{(k-1 / 2)} .
$$

2) $i \in \mathcal{D}^{(k)}, j \in \mathcal{B}^{(k-1)}$. By (2), $x_{j}^{(k-1 / 2)}$ must be strictly positive. This allows for both negative and positive variations $\delta x_{j}^{(k-1 / 2)}$. Calculations for a positive variation can be found in the previous section. For $\delta x_{j}^{(k-1 / 2)}<0$, (1) implies a decrease in state $j$ 's availability and therefore causes a new regime $\mathcal{D}^{\prime \prime}=\mathcal{D}^{(k-1)} \backslash\{j\}$ to occur at the very end of $\mathcal{D}^{(k-1)}$. $\mathcal{D}^{(k-1)}$ is then limited only by $\{j\}$, therefore (4) can be used to obtain its reduced duration $t^{(k-1)}+\delta x_{j}^{(k-1 / 2)} / f_{j}\left(\mathcal{D}^{(k-1)}\right)$. The remaining duration of $\mathcal{D}^{\prime \prime}$ is given by $\delta t^{\prime \prime}=t^{(k-1)}-\left(t^{(k-1)}+\right.$ $\left.\delta x_{j}^{(k-1 / 2)} / f_{j}\left(\mathcal{D}^{(k-1)}\right)\right)=-\delta x_{j}^{(k-1 / 2)} / f_{j}\left(\mathcal{D}^{(k-1)}\right)$. During $\delta t^{\prime \prime}$, all states $i \in \mathcal{D}^{(k-1)}$ are reduced by flow rates $f_{i}\left(\mathcal{D}^{\prime \prime}\right)$ instead of $f_{i}\left(\mathcal{D}^{(k-1)}\right)$. This varies the subsequent $x_{i}^{(k+1 / 2)}$ by $\delta x_{i}^{(k+1 / 2)}=\left(f_{i}\left(\mathcal{D}^{(k-1)}\right)-\right.$ $\left.f_{i}\left(\mathcal{D}^{\prime \prime}\right)\right) \delta t^{\prime \prime}$. Averaging sensitivities for negative and positive variations yields

$$
\begin{aligned}
\frac{\delta x_{i}^{(k+1 / 2)}}{\delta x_{j}^{(k-1 / 2)}=} & \frac{f_{i}\left(\mathcal{D}^{(k-1)} \backslash\{j\}\right)-f_{i}\left(\mathcal{D}^{(k-1)}\right)}{2 f_{j}\left(\mathcal{D}^{(k-1)}\right)} \\
& +\frac{f_{i}\left(\mathcal{D}^{(k)}\right)-f_{i}\left(\mathcal{D}^{(k)} \cup\{j\}\right)}{2 f_{j}\left(\mathcal{D}^{(k)} \cup\{j\}\right)},
\end{aligned}
$$

where positive denominators are ensured by (2) and (3). For $i \in \mathcal{D}^{(k)},\{j\}=\mathcal{B}^{(k-1)}$, it follows that $\mathcal{D}^{(k)}=\mathcal{D}^{(k-1)} \backslash\{j\}$ and $\mathcal{D}^{(k-1)}=\mathcal{D}^{(k)} \cup\{j\}$, which simplifies (7):

$$
\frac{\delta x_{i}^{(k+1 / 2)}}{\delta x_{j}^{(k-1 / 2)}}=\frac{f_{i}\left(\mathcal{D}^{(k)}\right)-f_{i}\left(\mathcal{D}^{(k-1)}\right)}{f_{j}\left(\mathcal{D}^{(k-1)}\right)}
$$

These results are now incorporated into the recursive update scheme for all nonzero rows $i \in \mathcal{D}^{(k)}$ of the approximate Jacobian

$$
\begin{aligned}
\frac{\partial x_{i}^{(k+1 / 2)}}{\partial x_{j}^{(-1 / 2)}} & =\sum_{l=1}^{n} \frac{\delta x_{i}^{(k+1 / 2)}}{\delta x_{l}^{(k-1 / 2)}} \frac{\partial x_{l}^{(k-1 / 2)}}{\partial x_{j}^{(-1 / 2)}} \\
& =\frac{\delta x_{i}^{(k-1 / 2)}}{\delta x_{j}^{(-1 / 2)}}+\sum_{l \in \mathcal{B}^{(k-1)}} \frac{\delta x_{i}^{(k+1 / 2)}}{\delta x_{l}^{(k-1 / 2)}} \frac{\partial x_{l}^{(k-1 / 2)}}{\partial x_{j}^{(-1 / 2)}}
\end{aligned}
$$

where the variational quotients are given by (7) or (8) and initial sensitivities are obtained from (5) or (6).

This calculation scheme also holds for the process' final sub-time step $K$. The final result $\partial \mathbf{x}^{(K+1 / 2)} / \partial \mathbf{x}^{(-1 / 2)}(=$ $\left.\partial \mathbf{x}^{(K)} / \partial \mathbf{x}^{(0)}\right)$ yields the sensitivities of all final process states with respect to the initial ones.

\section{OUTLOOK}

We presented a generalized representation of Daganzo's Cell Transmission Model, which allows for arbitrary cell connectivity and approximate sensitivity analysis.

Although no strict proof of the model's correctness in terms of the LWR theory is given, its strong structural analogy to the original CTM hopefully makes it a promising approach also from a more theoretical point of view. Due to its approximate differentiability, the model is well suited for application in a mathematical programming context.

We prepare to give experimental results on the model's applicability in the near future.

\section{REFERENCES}

[1] K. Ashok, Estimation and Prediction of Time-Dependent OriginDestination Flows, PhD Thesis, Massachusetts Institute of Technology, 1996.

[2] J. Bottom, Consistent Anticipatory Route Guidance, PhD Thesis, Massachusetts Institute of Technology, 2000.

[3] F. Crittin, M. Bierlaire, Solving the anticipatory route guidance generation problem using a generalization of secant methods, Conference Paper of 3rd Swiss Transport Research Conference, 2003.

[4] C.F. Daganzo, The cell-transmission model: A simple dynamic representation of highway traffi c, Trans. Res., vol. 28B(4), 1994, pp 269287.

[5] C.F. Daganzo, The cell-transmission model, part II: Network traffi c, Trans. Res., vol. 29B(2), 1995, pp 79-93.

[6] D. Helbing, A section-based queuing-theoretical traffic model for congestion and travel time analysis in networks, J. Phys. A: Math. Gen., 36, 2003, pp 593-598.

[7] M.J. Lighthill and J.B. Witham, On kinematic waves II. A theory of traffi c flow on long crowded roads, Proc. Royal Soc., A229, 1955, pp 317-345.

[8] M. Balmer, K. Nagel, and B. Raney, Large scale multi-agent simulations for transportation applications, J. of Intelligent Transport Systems, 8, 2004, pp 205-223.

[9] M. Papageorgiou, Optimierung: Statische, dynamische und stochastische Verfahren für die Anwendung, Oldenbourg Verlag, 1996.

[10] Y. Wang, M. Papageorgiou, Real-time freeway traffi c state estimation based on extended Kalman fi lter: a general approach, Trans. Res., vol. 39B, 2005, pp 141-167. 